\title{
Quantitative evaluation and analysis of adjustable potential of electric vehicle in demand response
}

\author{
Tiantian Qian a, ${ }^{\text {, }}$ Y Yaping Li, Xiaorui Guo and Jiantao Liu \\ China Electric Power Research Institute (Nanjing), Nanjing 210003, China \\ aqiantiantian@epri.sgcc.com.cn
}

Keywords: Electrically adjust the potential, electric car, Charge

\begin{abstract}
There is more and more electric vehicle users, and the electric vehicle load charging demand is also growing. It is increasingly necessary to study its reasonable charging strategy. This paper studies two controllable charging strategies, and quantitatively analyzes the charging power demand under this strategy. The results show that in the appropriate charging strategy that does not affect the mobility of the vehicle, the demand response potential of electric vehicles is considerable and can be used as a scheduling resource in demand response program.
\end{abstract}

\section{Introduction}

The development of electric vehicles involves national strategic issues such as environmental protection, energy security and industry development, which has been highly valued and strongly supported by governments.

According to data statistics, in 2020, China's electric vehicles will reach 5 million to 1000 million vehicles [1]. The power demand of electric vehicle will be particularly large, however, in the premise of meeting the charging demand of users, the charging mode of electric vehicle is very flexible, the charging load of which is controllable. This provides a very good controllable resource for the load control and demand response (DR) for the smart grid [2-4]. The Federal Energy Regulatory Commission (FERC) noted that, in order to specify the reasonable rate of transmission of DR in the wholesale market, and accurately measure and validate the demand side resources involved in the capacity market, DR potential assessments and predictions need to be provided to market operators. The dispatcher needs to know the schedulable flexible load that the electric vehicle cluster can provide at a particular time. So it is necessary to study the quantitative analysis of its control strategy and its schedulable demand response potential.

In the absence of the guidance and stimulation of an effective demand response mechanism, according to their living habits and travel requirements, the charging strategy of electric vehicle users is random charging strategy. Under the random charging strategy, the users only need to consider their driving needs, there is no need to consider the cost-effectiveness of price changes, so the users' satisfaction is highest. In the natural state, the charging peak time of electric vehicle load is roughly in the 16:00 19:00, which is the rush hour, overlap with the peak power consumption of the grid. As the electric vehicles have the energy storage properties, if take the appropriate charging strategy, will make the electric vehicle to reduce the charging time in the peak time and will not affect its mobility.

Most of the existing literatures have [5-7] studied the charging control strategies of large-scale electric vehicles after they connected to the power grid. However, there is little literature on the quantitative analysis of the specific schedulable demand response potential under the detailed charging strategy. In this paper, several EV potential assessment scenarios are designed. Furthermore, the potential assessments of the demand response potential of electric vehicles under the corresponding scenarios are studied. 


\section{Quantitative Evaluation of the Schedulable Potential of Electric Vehicles}

The quantitative analysis of charging load of electric vehicle cluster needs specific vehicle driving conditions: the start-stop time of the driving activity, driving distance, the start-stop time of the parked activity, the power exchanged with the power grid. As there has not been able to get the related information of current domestic electric vehicle, so use the electric vehicle activity statistics provided by United States NHTS for analysis.

For a single electric vehicle, its activity serial number $\mathrm{i}=1,2,3, \cdots$, the active event expression is as follows:

$$
\operatorname{activity}(i)=\left\{\begin{array}{l}
\text { charging } \\
\text { parked } \\
\text { driving }
\end{array}\right.
$$

The electric vehicle model is Nissan leaf, the statistics information is shown in Table 1.

Table 1. Three Scheme comparing.

\begin{tabular}{ccccccc}
\hline Vehicle ID & State & Start time & End time & Distance $(\mathrm{m})$ & P_max $(\mathrm{W})$ & Location \\
\hline 21 & Charging & $0: 00: 00$ & $7: 35: 00$ & -1 & 1440 & Home \\
\hline 21 & Driving & $7: 35: 00$ & $8: 00: 00$ & 16093.44 & -1 & -1 \\
\hline 21 & Parked & $8: 00: 00$ & $8: 05: 00$ & -1 & -1 & Personal Obligations \\
\hline 21 & Driving & $8: 05: 00$ & $8: 20: 00$ & 16093.44 & -1 & -1 \\
\hline 21 & Charging & $8: 20: 00$ & $0: 00: 00$ & -1 & 1440 & Home \\
\hline
\end{tabular}

\subsection{The charging load model of electric vehicle in the random charging mode}

The calculation of the charging load of electric vehicle is based on the days, and the time interval is accurate to minutes. The total charge load of the $\mathrm{i}$-th minute is the sum of the charge loads for all vehicles at this time.

$$
P_{i}=\sum_{n=1}^{N} P_{n, i}
$$

In the formula, $P_{i}$ is the total charge power of the $\mathrm{i}$-th minute, $\mathrm{N}$ is the total amount of electric vehicles, $P_{n, i}$ is the charging power of the nth vehicle in the i-th minute.

\subsection{Charge load model of electric vehicle in controllable charge mode}

In addition to the above statistics, it is also necessary to understand the state of charge (SOC) data in each state of the electric vehicle, to develop a reasonable charging strategy.

The calculation model of SOC:

$$
\mathrm{SOC}=\frac{E_{r}}{E_{T}}
$$

In the formula, $E_{r}$ is the remaining energy available for electric vehicle battery, $E_{T}$ is the total release energy of electric vehicle battery. $E_{r}$ can be calculated by the following formula:

$$
E_{r}=E_{T}-E_{c o n}+E_{c h}
$$

In the formula, $E_{c o n}$ is the energy consumed by driving an electric vehicle, $E_{c h}$ is the energy supplemented by charging an electric vehicle.

\subsection{The Energy Consumption Model of Electric Vehicle in Driving State}

The research on energy consumption model of electric vehicle at home and abroad is commonly based on the speed - time series of energy consumption calculation model. The input of the model is a velocity-time series of operating conditions, and the output of the model is the energy consumption of electric vehicle, the unit of which is $\mathrm{kWh}$. According to the driving situations on the city road of electric vehicle, the driving state of electric vehicle can be divided into idling, uniform velocity, acceleration, deceleration four states. The mathematical expression of its energy consumption model is as follows:

$$
E_{c o n}=E_{t} / \eta_{m} / \eta+E_{a}+E_{b} \cdot \eta_{b}
$$


In the formula, $E_{t}$ is the energy consumed by the electric vehicle driving force in a uniform or accelerated state. $E_{a}$ is the energy consumed by electric vehicle accessories in the idle speed state. $E_{b}$ is the energy consumed by the electric vehicle braking force in the deceleration state. $\eta_{m}$ is the mechanical transmission efficiency of electric vehicle transmission system. $\eta$ is the efficiency of electric vehicle motor. $\eta_{b}$ is the recovery efficiency of electric vehicle braking energy. Using the above mathematical model to calculate the energy consumption of vehicle requires more parameters, unfavorable to engineering calculation. Therefore, this article uses the average energy consumption per kilometer driving mileage to calculate the energy consumption of electric vehicles. the expression is as follows:

$$
E_{c o n}=E_{a v} \cdot l
$$

In the formula, $E_{a v}$ is the average energy consumption of electric vehicle per kilometer, $l$ is the driving mileage.

\subsection{The charging power model of electric vehicle in the driving state}

The start time of the demand response is $t_{s}$, the stop time is $t_{e}$, the charging power of the electric vehicle in the demand response period is

$$
P_{D R, c h}=\frac{E_{D R, c h}}{t_{s}-t_{e}}
$$

Under different charging strategies, have different $E_{D R, c h}$ mathematical models. The charging strategy designed in this paper, the principle of which is to reduce the amount of charge during the demand response time, but at the same time to ensure that the user's normal driving needs.

The charging period of the electric vehicle intersects the demand response period, shown as Figure 1. Remove the charging behavior of the intersection with the demand response, calculate the SOC of the electric vehicle. The algorithm is as follows:

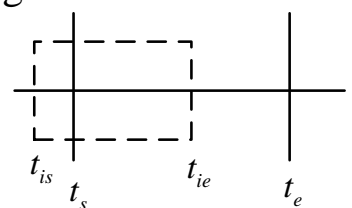

(a)

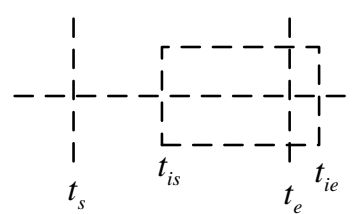

(c)

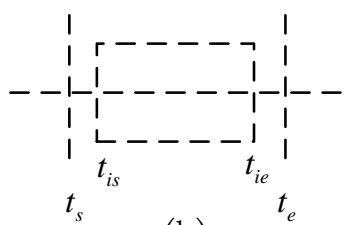

(b)

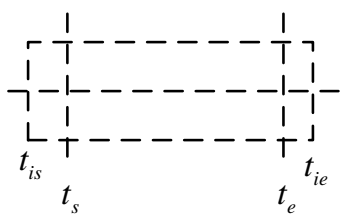

(d)

Figure 1. The relationship between demand response period and the charging time of electric vehicle.

Assume that the $\mathrm{i}$-th activity of the electric vehicle is a charging activity, there are four scenarios for the period in which the charging activity intersects the demand response. In figure (a), remove the intersection with the demand response period, the charging time is $\Delta t=t_{s}-t_{i s}$. In figure (b), remove the intersection with the demand response period, the charging time is 0 . In figure (c), remove the intersection with the demand response period, the charging time is $\Delta t=t_{i e}-t_{e}$. In figure (d), remove the intersection with the demand response period, the charging time is $\Delta t=\left(t_{s}-t_{i s}\right)+\left(t_{i e}-t_{e}\right)$.

Remove the charging activity that intersects the demand response period, the charging capacity of activity(i) are respectively $E_{i c}=P_{i c} \cdot\left(t_{s}-t_{i s}\right), E_{i c}=P_{i c} \cdot 0, E_{i c}=P_{i c} \cdot\left(t_{i e}-t_{e}\right), E_{i c}=P_{i c} \cdot\left(\left(t_{s}-t_{i s}\right)+\left(t_{i e}-t_{e}\right)\right)$. Hereby, the SOC sequence of the electric vehicle is calculated.

Scenario one: If the minimum SOC of electric vehicle within 24 hours is less than the safe minimum threshold 0.2 , the owner needs to charge the vehicle until the minimum SOC reaches 0.2. This part is user's rigid demand. 


$$
E_{D R, c h}=\left(0.2-S O C_{\text {min }}\right) \cdot E_{T}
$$

The average charging power of the demand response period is

$$
P_{D R, c h}=\frac{\left(0.2-S O C_{\min }\right) \cdot E_{T}}{t_{s}-t_{e}}
$$

The power demand calculation flow chart of scenario one is shown as Figure 2.

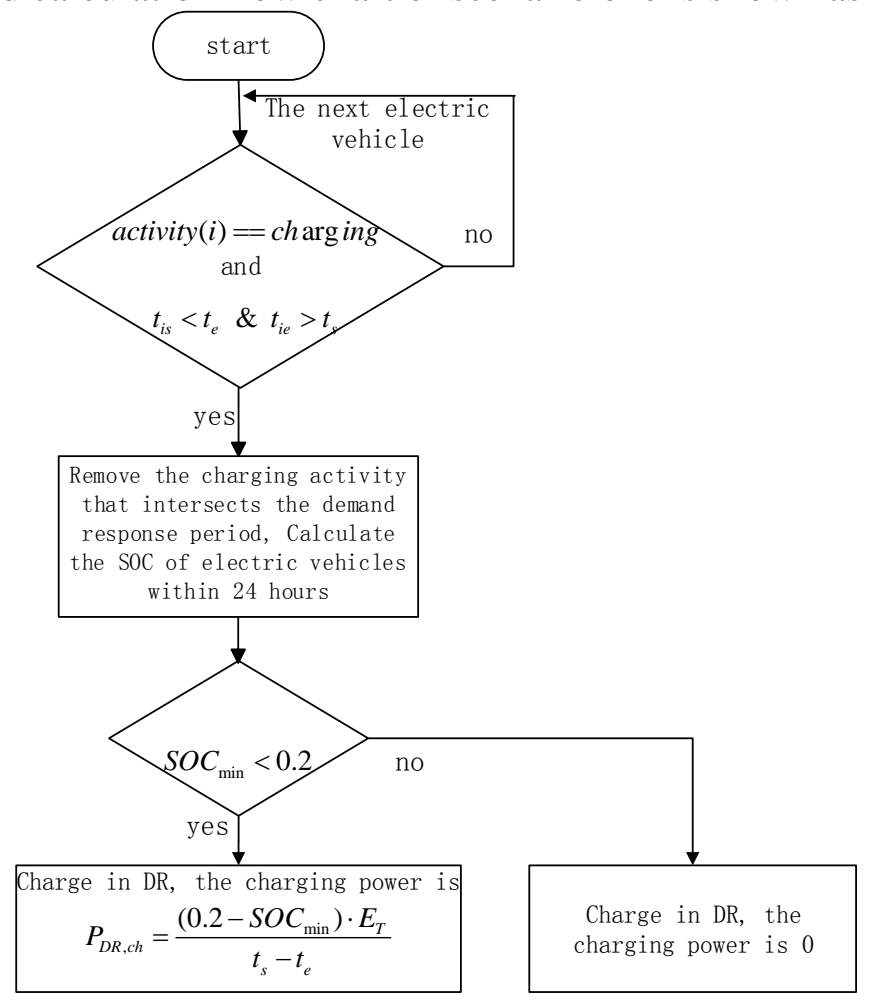

Figure 2. The calculation flow chart of scenario one

Scenario two: consider the user's "mileage anxiety" mentality, some users will choose to continue charging in the desired way during the demand response period (including the users in scenario one that charge to 0.2 and $S O C_{\text {min }}(i)$ is greater than 0.2 , but worry about the next mileage).

In order to quantitative analysis, choose the users whose activity(i), which intersect with DR, is charging activity and afterwards no charge activity but driving activity as "Range anxiety" users.

In this situation, the power calculation of the electric vehicle in the demand response period is shown as Figure 3 in the following flowchart:

\section{Case study}

Using python language and taking electric vehicle user driving statistics provided by NHTS, programming scenario 1 and scenario two, calculate the charging power curve of the electric vehicle.

Based on scenario one:

The DR period is from 17:00 to 19:00, the power demand comparison based on scenario one of 2000 electric vehicles in the charge control state and uncontrolled state is shown as below. The power demand can be reduced from near 1.5 MW to lower than 0.1 MW in DR period. 


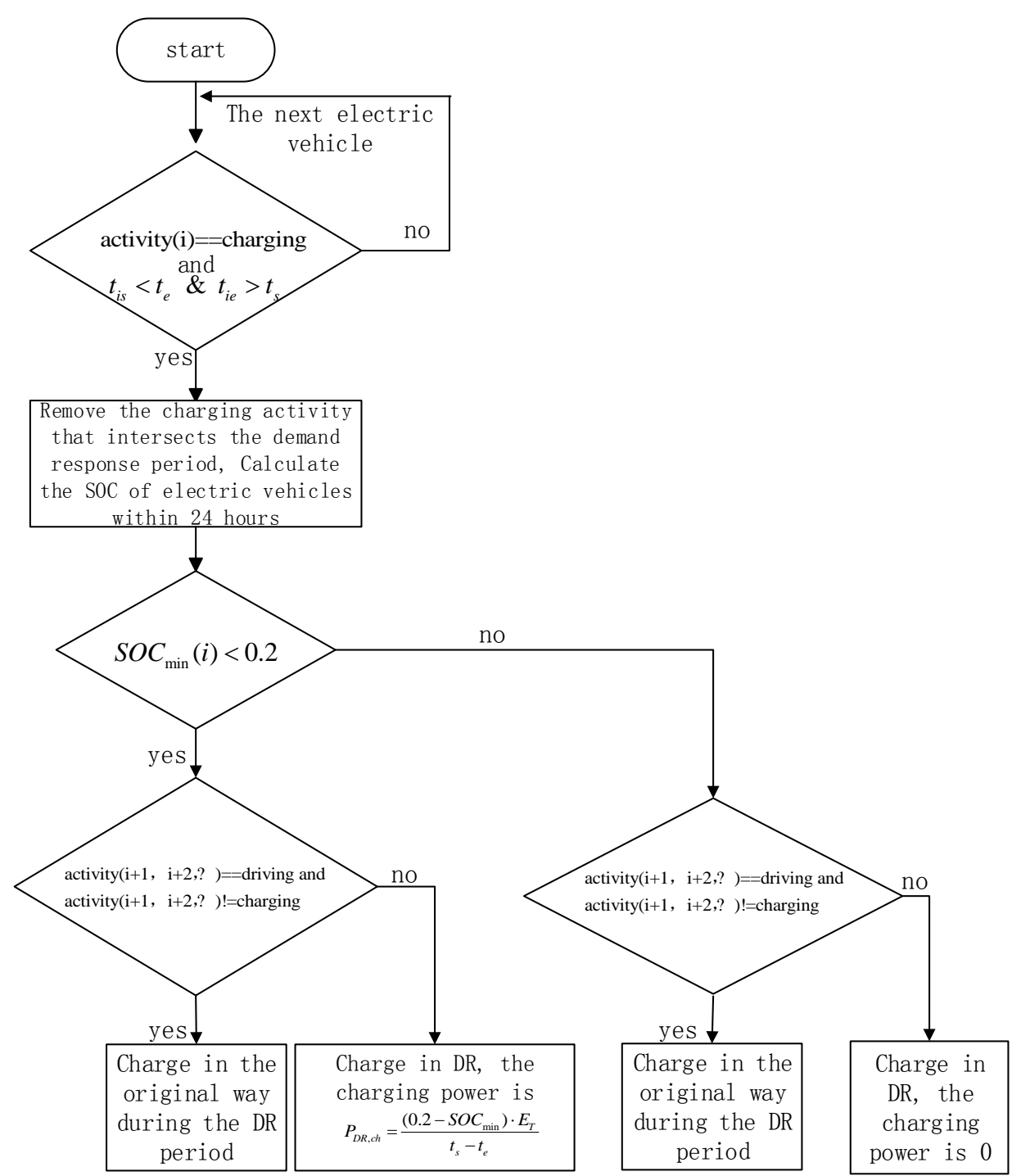

Figure 3. The calculation flow chart of scenario two

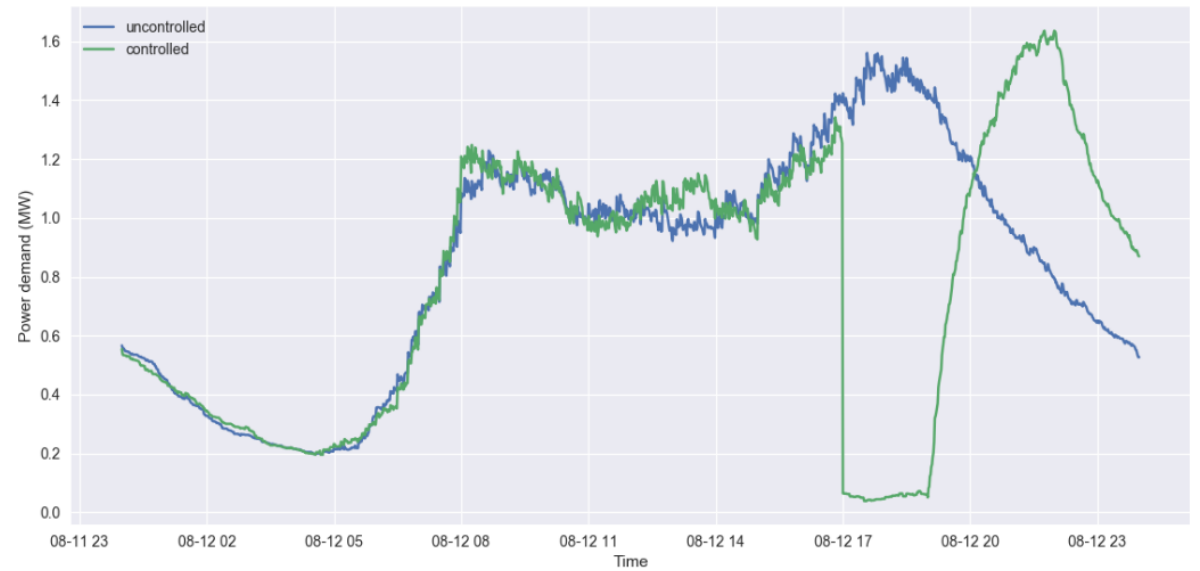

Figure 4. The power comparison chart of scenario one

Based on scenario two:

The DR period is from 17:00 to 19:00, the power demand comparison based on scenario two of 2000 electric vehicles in the charge control state and uncontrolled state is shown as below. The power demand can be reduced from near 1.5 MW to near 0.2 MW in DR period. 


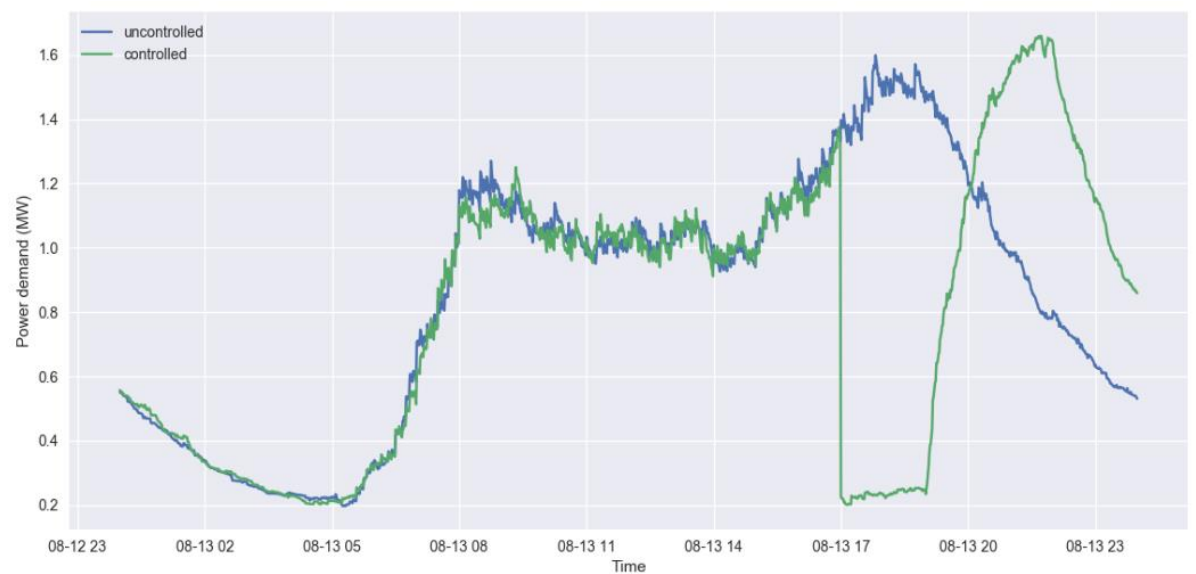

Figure 5. The power comparison chart of scenario two

\section{Conclusion}

This paper quantitatively studies the charging power demand of electric vehicles in uncontrollable state and controllable state. This study shows that with proper controlled charging strategies without compromising the mobility of electric vehicles, the charging loads can be reduced to a great extent during peak demand periods. This suggests that electric vehicles are highly flexible loads.

\section{Acknowledgments}

This work was financially supported by National Key Research and Development Program of China (2016YFB0901101).

\section{References}

[1]. WANG Dai, GUAN Xiaohong, WU Jiang and etc, Vehicle driving pattern based modeling and analysis of centralized charging/discharging strategy for plug-in electric vehicles [J], Power System Technology, 2014, 38(9): 2322-2327.

[2]. HU Zechun, SONG Yonghua, XU Zhiwei and etc, Impacts and utilization of electric vehicles integration into power systems [J], Proceedings of the CSEE, 2012, 32(4): 1-10.

[3]. Tian Liting, Zhang Mingxia, Wang Huanling, Evaluation and solutions for electric vehicles' impact on the grid [J], Proceedings of the CSEE, 2012, 32(31): 43-49.

[4]. Zhao J, Wen F, Yang A, et al. Impacts of electric vehicles on power systems as well as the associated dispatching and control problem [J]. Automation of Electric Power Systems, 2011, 14: 2-9.

[5]. Shaoyun G, Long W, Hong L, et al. An optimization model of peak-valley price time-interval considering vehicle-to-grid[J]. Power System Technology, 2013, 37(8): 2316-2321.

[6]. Xu Z, Hu Z, Song Y, et al. Coordinated charging strategy for PEV charging stations based on dynamic time-of-use tariffs[J]. Proceedings of the CSEE, 2014, 34(22): 3638-3646.

[7]. Liu Dongqi, Wang Yaonan, Shen Yongpeng, Research of V2G Smart Charging Station Control Strategy Using T-S Fuzzy Controller [J], TRANSACTIONS OF CHINA ELECTROTECHNICAL SOCIETY, 2016, 31(2): 206-214. 